\title{
Adquisición y desarrollo del lenguaje en bilingües familiares: primeros datos de una investigación *
}

\section{Ignasi Vila \\ Humbert Boada y Miquel Siguan **}

Universidad Central de Barcelona

$\mathrm{El}$ reconocimiento «oficial» de las lenguas vernáculas en las divèrsas nacionalidades de nuestro Estado y su consiguiente implantación en los niveles de enseñanza subrayan la urgencia de contar con un corpus de investigación que avale y facilite esa implantación. Los autores de este artículo tienen ya una trayectoria importante en la constitución y fomento de ese cuerpo de investigación, y este estudio constituye una aportación más en esa línea.

* Esta investigación se inscribe en el plan de investigaciones del ICE de la Universidad de Barcelona sin cuy,o apoyo no hubiera sido posible este trabajo. Queremos agradecer, igualmente, la ayuda de Isabel Mas en la transcripción del material de vídeo.

** Dirección de los autores: Universidad Central de Barcelona. Facultad de Filosof́a y C.C.E.E. Dpto. Psicologia General. Barcelona. 
El primer sistema comunicativo que el niño utiliza está basado, primordialmente, en procedimientos no verbales. En cierto momento, un sistema lingüístico autónomo irá sustituyendo progresivamente gran parte de dichos procedimientos en la consecución de objetivos comunicativos. Este sistema lingüístico presenta diversas dificultades en su aprendizaje, recibiendo su estudio distintos tratamientos a lo largo de los últimos 20 años. Sin embargo, quedan muchos aspectos sin resolver.

Son muchos los factores que ayudan a ello. Entre otros, tenemos que tanto la sintaxis como la semántica predicen de forma válida la dificultad que tiene el niño en aprender un morfema (Brown, 1973). Ello no es extraño, ya que muchos de los aspectos gramaticales son también aspectos del significado. Por tanto, la dificultad en la adquisición de una flexión vendrá dada por un conjunto de factores semánticos y gramaticales, siendo difícil determinar la contribución de cada uno.

Slobin (1973) aporta un método para separar los efectos de la complejidad semántica y la complejidad gramatical, a partir de la observación de dós niños que aprendían simultáneamente húngaro y serviocroata. Algunos significados expresados en castellano a través de preposiciones locativas, se expresan en húngaro por inflexiones sobre el nombre, requiriendo en servio-croata, además una preposición. Los dos niños utilizaron antes las inflexiones en húngaro, que la forma completa en servio-croata, mostrando que conocían antes el concepto que la forma en que se expresaba en esta lengua.

Dicho estudio muestra que las personas que adquieren simultáneamente dos o más lenguas constituyen un caso singular para el estudio de los procesos de adquisición del lenguaje, cual es el caso de los bilingües familiares que realizan desde el nacimiento el aprendizaje de dos lenguas.

La literatura sobre estos bilingües comienza en Ronjat (1913) y es continuada hasta nuestros días por distintos autores. Varios de ellos, sobre todo al inicio, son lingüistas que analizan el lenguaje de su propio hijo (Leopold, 1939, 1947, 1949), poseyendo estos sujetos una educación linguística cuidada. Un caso distinto es el de Tabouret-Keller (1969), en el que el sujeto pertenecía a un ambiente socio-cultural bajo. Debe citarse también a Ruke-Dravina (1967), y los más recientes de Swain (1976) y de la Escuela de Roma (Volterra y Taeschner, 1978, Titone, 1980).

Básicamente, dichos estudios coinciden en postular la existencia de un sistema único lexical en los primeros estadios de la adquisición del lenguaje, siendo su desarrollo semejante al del niño monolingüe. Hasta el momento no poseemos estudios de este tipo eh el área lingǘrstica ca- 
talana, que atraviesa desde siglos, y acentuado últimamente, un fuerte contacto con el castellano. En consecuencia, nuestra intención es el estudio de la adquisición del lenguaje en 3 niños bilingües familiares y dos monolingües, uno catalán y otro castellano.

La presente exposición se limita a exponer unos primeros datos sobre el primer estadio del sistema lingǘrstico de uno de nuestros sujeros, María del Mar, referidos a la existencia de un solo código lingüístico.

\section{Método}

El sujeto es una niña, bilingüe familiar, padre de habla catalana y madre de habla castellana. El nivel socio-cultural es medio-alto. La niña asiste a una guardería, cuya lengua vehicular es el catalán.

Se han efectuado sesiones de registro de 30', desde los 6 meses con procedimientos de video-tape. En este momento su edad es de 25 meses. El registro se hace en el hogar y en presencia de un adulto como mínimo, intentando que no haya un gran intervalo entre la presencia de cada uno de los cónyuges. Los registros en video-tape se complementan con registros personales directos durante el intervalo entre los 15 días.

\section{Resultados}

Los dividiremos en dos partes. En un primer momento, María del Mar utiliza palabras provenientes de los dos léxicos o mixtas sin que parezca conocer la relación que existe entre equivalentes. En un segundo momento se da cuenta de la existencia de equivalentes, es decir que un mismo objeto, propiedad $\mathbf{u}$ acción, puede tener dos formas equivalentes de significación.

El léxico utilizado por María del Mar en el primer momento se corresponde con el que se encuentra en investigaciones precedentes (Nelson, 1973; Ninio y Bruner, 1978; Howe, 1981), destacando un elevado número de sustantivos, nombres propios y acciones con una referencia clara. Igualmente aparecen expresiones referidas a contextos ritualizados socialmente como la despedida, el saludo, etc. y algunas propiedades, así como palabras-función.

Respecto al código del cual proceden obtenemos un elevado porcentaje de palabras en castellano, siendo bastante reducido el número de palabras procedentes del catalán. Prácticamente no hay equivalentes y los que aparecen son utilizados de forma diferente por María del Mar. 
Tabla I

M.a del Mar $(2 ; 0,12)$

\begin{tabular}{|c|c|c|c|c|c|c|}
\hline \multicolumn{3}{|c|}{ CASTELLANO } & \multicolumn{2}{|c|}{ CATALAN } & \multicolumn{2}{|c|}{ MIXTAS } \\
\hline $\begin{array}{r}\text { agua } \\
\text { pelota } \\
\text { quema } \\
\text { mío } \\
\text { beber } \\
\text { más } \\
\text { manzana } \\
\text { mala } \\
\text { plátano } \\
\text { pescado } \\
\text { mañana } \\
\text { huevo } \\
\text { dame } \\
\text { cuarto } \\
\text { azúcar } \\
\text { todo } \\
\text { casa } \\
\text { guapa }\end{array}$ & $\begin{array}{l}\text { adiós } \\
\text { nariz } \\
\text { oreja } \\
\text { mía } \\
\text { dormir } \\
\text { caballo } \\
\text { cepillo } \\
\text { pato } \\
\text { zapato } \\
\text { ven } \\
\text { comer } \\
\text { otro } \\
\text { conejo } \\
\text { este } \\
\text { abre } \\
\text { silla } \\
\text { vaso } \\
\text { gato }\end{array}$ & $\begin{array}{l}\text { así } \\
\text { tren } \\
\text { leotardo } \\
\text { ojo } \\
\text { diente } \\
\text { servilleta } \\
\text { gallina } \\
\text { indio } \\
\text { naranja } \\
\text { «buenas noches» } \\
\text { puedo } \\
\text { lobo } \\
\text { hace } \\
\text { magnetofón } \\
\text { carne } \\
\text { tenedor } \\
\text { cuchillo } \\
\text { "cumpleaños feliz» }\end{array}$ & $\begin{array}{l}\text { té } \\
\text { nina } \\
\text { liuna } \\
\text { qui } \\
\text { pà } \\
\text { prou } \\
\text { llum } \\
\text { més } \\
\text { panxa } \\
\text { gall } \\
\text { vina } \\
\text { sabata } \\
\text { aixó } \\
\text { cullera } \\
\text { pitet } \\
\text { tornaquet } \\
\text { mà } \\
\text { tonto (1) } \\
\text { vermell } \\
\text { groc } \\
\text { ara } \\
\text { anem } \\
\text { poma }\end{array}$ & 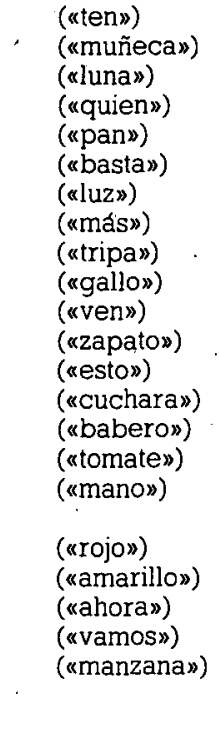 & $\begin{array}{l}\text { sí } \\
\text { mira } \\
\text { aquíací } \\
\text { fábrica } \\
\text { pupa } \\
\text { coco } \\
\text { jersey } \\
\text { no } \\
\text { sol } \\
\text { y/i } \\
\text { café } \\
\text { tu } \\
\text { boca } \\
\text { biberón } \\
\text { ahr } \\
\text { Mar } \\
\text { Javier } \\
\text { Marta } \\
\text { Patxi }\end{array}$ & $\begin{array}{l}\text { guau-guau } \\
\text { pandereta } \\
\text { "ya está»/«ja está» } \\
\text { mamá } \\
\text { espera } \\
\text { sopa } \\
\text { aparta } \\
\text { tapa } \\
\text { pipí } \\
\text { es } \\
\text { hola } \\
\text { que } \\
\text { papa } \\
\text { vaca } \\
\text { rana } \\
\text { Nacho } \\
\text { Mañu } \\
\text { Ana } \\
\text { Ariadna. }\end{array}$ \\
\hline
\end{tabular}

Las palabras en negrita son equivalentes

(1) fonéticamente, claramente en catalán 
En este estadio, María del Mar, rechaza la posibilidad de designar un objeto de forma diferente al nombre que tiene aprendido.

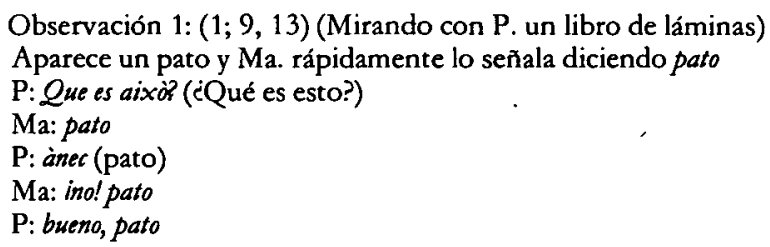

Posee algún nombre equivalente, en términos del adulto, pero los utiliza de forma diferente.

Observación 2: $(1 ; 9,13)$ (Mirando con M. un libro de láminas)

M: mira, mira, María del Mar (señala una manzana)

Ma: (señala la manzana, sonríe)

$\mathrm{M}:$ ZQué es esto?

Ma: manzana

(En la mesa, comiendo, M trae una manzana)

M: mira, María del Mar, traigo una manzana

Ma: (señalando) poma («manzana») .

$\mathrm{M}$ : una manzana

Ma: no, poma

En este caso poma se refiere a la fruta comestible y manzana a la representación gráfica. Volterra y Taeschner (1978) obtienen los mismos resultados en niños qie aprendían simultáneamente italiano y alemán, señalando con ello la dependencia del contexto en que una palabra es incorporada en los primeros momentos de la adquisición del lenguaje.

Al igual que en los niños monolingües hemos observado fenómenos de «sobreextensión», así como de confusión en la utilización de términos deicticos.

Observación $3:(1 ; 11,1)$

$\mathrm{M}$ escribe en un papel y Ma contemplándolo extiende el brazo hacia el lápiz queriéndolo coger. A la vez exclama ara tu, ara tu, M le deja el lápiz y Ma dibuja.

En un segundo, María del Mar, parece conocer que las mismas cosas son designadas de forma distinta por los adultos que conviven con ella.

Observación $4:(2 ; 0,12)$

(Mirando con uno de los experimentadores un cuento, aparece un pato)

E: (señala el pato) LQué es esto?

Ma: (vuelve la cabeza hacia $P$ y ríe) pato

P: (mirando a $\mathrm{Ma}$ ) ànec

Ma: pato

P: ànec ("patom)

(Ma vuelve a centrarse en el cuento)

E: (señala el pato) $2 Q$ Qué es esto?

Ma: ànec

E: ¿Qué es?

Ma: pato

E: pato, si, pato

Ma: (mirando a E) ànec

E: (señala el pato) LQué es esto?

Ma: pato, ànec 
Analizando el aumento de vocabulario a partir de dicho momento y durante el perfodo de un mes tenemos la Tabla II.

En ella se observa un equilibrio mayor de palabras provenientes de ambos códigos, así como la incorporación de un buen número de equivalentes. También es de destacar que un $50 \%$ del total de palabras incorporadas tengan la misma forma en catalán y castellano.

Sin embargo, no podemos afirmar que Marfa del Mar tenga ya conciencia de la existencia de dos léxicos en su ambiente familiar, ya que en determinadas ocasiones se empeña en designar un objeto o suceso a través del nombre que posee en su sistema, negando la posibilidad que pueda recibir otro nombre.

\section{Observación 5: $(2 ; 1,15)$}

(Haciendo con $\mathrm{M}$ un muñeco formado por piezas. Están casi al final y $\mathrm{Ma}$ coge el sombrero.)

$\mathrm{M}$ : źy eso qué es? '́el gorro?

Ma: (extiende todo el cuerpo hacia $M$ ) ino!

M: iqué es esto?

Ma: barret («sombrero»)

M: iab! un barret

Ma: $s i$

$\mathrm{M}:$ ino es un gorro?

Ma: $n o$

M: ab, bueno

Incluso equivalentes que incorpora parecen tener un campo semántico diferente.

\section{Observación 6: $(2 ; 1,15)$}

(Mirando con $\mathrm{M}$ un juego de fichas. Aparece una tortuga y encima de ella una más pequeña)

M: (señala la tortuga grande) Una tortuga grande

(señala la tortuga pequeña) ¿ $Y$ ésta cómo es?

Ma: tortuga

$\mathrm{M}:$ by cómo es esta tortuga?

Ma: petita, petita ("pequeña, pequeña»)

M: es pequeña

(señala la tortuga grande) $2 Y$ ésta cómo es?

Ma: pequeña

M: ¿cómo es esta tortugal

Ma: pequeña

$\mathrm{M}$ : (señalando altemativamente) ésta es pequeña y ésta es grande.

Finalmente, señalar que utiliza todo su vocabulario para dirigirse a los adultos, independientemente de la lengua de cada uno.

\section{DISCUSION}

Los resultados obtenidos, hasta el momento, parecen concordar con los de las investigaciones realizadas anteriormente. En el terreno de la comprensión, María del Mar, no tiene una dificultad especial para adecuarse a los requerimientos, provisiones de información, etc. dirigi- 
TABLA II

M.a del Mar $(2 ; 1,15)$

\begin{tabular}{|c|c|c|c|}
\hline CASTELLANO & \multicolumn{2}{|c|}{ CATALAN } & MIXTAS \\
\hline $\begin{array}{l}\text { quien } \\
\text { vamos } \\
\text { cerdito } \\
\text { dinero } \\
\text { bolsillo } \\
\text { sucio } \\
\text { botón } \\
\text { caído } \\
\text { suelo } \\
\text { pollo } \\
\text { «muy mal» } \\
\text { llora } \\
\text { ahora } \\
\text { pequeña } \\
\text { trapo } \\
\text { fuera } \\
\text { para } \\
\mathrm{N}=17\end{array}$ & $\begin{array}{l}\text { meva } \\
\text { aixeca't } \\
\text { anec } \\
\text { trancat } \\
\text { anat } \\
\text { meu } \\
\text { gat } \\
\text { butxaca } \\
\text { brut } \\
\text { peix } \\
\text { pruna } \\
\text { banyes } \\
\text { puc } \\
\text { mico } \\
\text { blau } \\
\text { passat } \\
\text { barret } \\
\text { cua } \\
\text { pilota }\end{array}$ & $\begin{array}{l}\text { («mía) } \\
\text { («levántate») } \\
\text { ("pato») } \\
\text { («roto») } \\
\text { ("ido») } \\
\text { ("mio») } \\
\text { ("gato») } \\
\text { ("bolsillo») } \\
\text { ("sucio») } \\
\text { ("pez») } \\
\text { ("ciruela») } \\
\text { («cuernos") } \\
\text { ("puedo») } \\
\text { ("mono») } \\
\text { ("azul») } \\
\text { ("pasado») } \\
\text { ("gorro») } \\
\text { ("cola») } \\
\text { ("pelota») }\end{array}$ & $\begin{array}{l}\text { pata (1) } \\
\text { bota } \\
\text { tenis } \\
\text { gracias/gracies } \\
\text { raqueta } \\
\text { Merche } \\
\text { M. Jesús } \\
\text { Joaquín } \\
\text { Teo } \\
\text { cole } \\
\text { trompa } \\
\text { barba } \\
\text { tortuga } \\
\text { vela } \\
\text { borra } \\
\text { yo/jo (2) } \\
\text { Luis } \\
\mathrm{N}=17\end{array}$ \\
\hline
\end{tabular}

Todas las palabras en negrita corresponden a equivalentes.

(1) forma utilizada por el padre al hablar catalán. En cat pota

(2) forma confusa entre cat/cast, por la forma en que se utiliza jo por la

población de Barcelona. 
dos en una u otra lengua. En el terreno de la producción parece poseer un único sistema lexical que incluye palabras de ambos códigos, siendo su desarrollo semejante al del niño monolingüe.

Junto a este desarrollo, aparece un fenómeno de relevancia en el desarrollo del lenguaje en los bilingües familiares: la utilización de términos equivalentes para designar un mismo objeto o suceso. Piaget (1923) y Vigotsky (1934) observaron que los niños consideraban el nombre de un objeto como una propiedad intrínseca e invisible del objeto. Estudios posteriores (Osherson y Markman, 1975; Berthoud1978), muestran que es entre los 4 y $7 / 8$ años, con variaciones importantes según la tarea, cuando el niño toma conciencia de las dimensiones lexicales del lenguaje. Sin embargo, la mayoría de autores que han estudiado el bilingüismo familiar sitúan la conciencia de la existencia de los dos léxicos y, consecuentemente, la posibilidad de nombrar un mismo objeto a través de signos distintos, en un momento bastante anterior. Slobin (1978) cita el caso de su hija, Heida, la cual poseía una conciencia metalingüistica notable con respecto a los aspectos semánticos del lenguaje a la edad de tres años, coincidiendo en ello con un buen número de autores. En nuestro caso, las primeras observaciones de distinción de los dos léxicos las observamos alrededor de los dos años, con un MLU (Mean Lenght of Utterance o longitud media de la frase) de 2, lo cual no implica, evidentemente, una conciencia metalingüística, pero sí un camino que la inicia.

Analizando con más detenimiento las palabras equivalentes que incorpora podemos establecer la siguiente clasificación:

a) palabras que ya utilizaba, pero que posefan un campo semántico diferente (poma/manzana).

b) palabras con una referencia clara a su actividad, tanto por el objeto como por la relación que designan (pilota, meu)

c) palabras directamente relacionadas con contextos de interacción con el adulto (vamos).

Tomadas en conjunto, las observaciones ponen de relieve la forma de la educación bilingüe en la familia, así como la importancia del adulto en el aprendizaje del lenguaje como "soporte pedagógico" respecto al cual basculan las hipótesis y estrategias del niño. Tanto el padre como la madre se dirigen a María del Mar en su propia lengua, sin hacer mezclas lingǘsticas. Sin embargo, ambos hacen una excepción: cuando María del Mar produce una palabra (en catalán o castellano) sin tener el mismo significado que tiene para el adulto o es una palabra recién incorporada, respetan en su propio lenguaje el código utilizado por la niña. Una vez que dicho significado, a su entender, está bien establecido intentan que María del Mar aprenda el equivalente. 
Las siguientes observaciones muestran parte del proceso.

Observación $7:(2 ; 1,15)$

(Ma comienza a nombrar los colores. M y Ma colocan tres fichas, cada una de forma y color distinto, en sus lugares correspondientes. Una vez situadas el diálogo es el siguiente.)

M: ¿Cuál es el vermelR («Cíál es el rojo?)

Ma: este (señala el verde)

$\mathrm{M}$ : inoi ieste es el verd! es el verde

¿Y el blau? ( $¿ Y$ el azul?)

Ma: (señala el azul) aquí.

M: ¿'Y el vermell?

Ma: (señala el rojo?) aqui

$\mathrm{M}:\{Y$ el verd?

Ma: (señala el verde) aqui

$\mathrm{M}:\{Y$ el vermell?

Ma: (señala el azul) aquí

M: ino!

Observación $8:(2 ; 0,5)$

Ma comienza a decir la palabra azúcar (en catalán sucre). (Jugando con P. "como sís prepararan café.)

P: (señala el azúcar) Que es això? (“¿ंQué es estoß»))

Ma: azicar

P: ל̌l azuican?

(extiende una cuchara $\mathrm{Ma}$ ) Vols una mica? ("iQQuieres un poco?»)

Ma: (señala la taza de P) papa

P: (se «pone» azúcar) pel papa («para papá)

(extiende la cuchara hacia la taza de Ma) Ara posarem axuicar. Quentes en vols? («Ahora pondremos azúcar. ¿Cuántas quieres?)

\section{Observación 9: $(2 ; 0,15)$}

(Mirando con M un libro de láminas de dibujo)

$\mathrm{M}$ : (señala un pez) \&Qué ban ido a comprar aqui?

Ma: peix ("pez»)

$\mathrm{M}:$ ZQué es eso?

Ma: peix

M: ipeix? Aqui ban ido a comprar pescado para cenar

Ma: iab!

M: (señala otro pez) ¿Y esto?

Ma: peix

En resumen: María del Mar se encuentra en un período de transición en la distinción de dos códigos lexicales. Èn este periodo aparecen. procesos semejantes al desarrollo del niño monolingüe, pero aparecen también estrategias particulares. Su estudio puedè ayudar, como señalábamos al comienzo, a explicitar con más claridad procesos subyacentes a la adquisición del lenguaje que necesitan aún numerosas investigaciones. 


\section{Referencias}

Berthoud-Papandropoulou, I.: An experimental study of children's ideas about language, (en) A. Sinclair, R. J. Jarvella y W. J. M. Levelt (eds.) The Child's Conception of Language. Berlin, Springer. 1978.

Brown, R. A first language. Cambridge, Mass., Harvard Univ. Press. 1973.

BRuner, J. S.: The formats of language acquisition. American Joumal of Semiotics, 1982, 1(2), en prensa.

Howe, C.: Acquiring language in a conversational context. London, Academic Press. 1981.

Leopold, W. F.: Speech Development of a Bilingual Child: a linguist's record. Evanston, Northwestern Univ. Press, 1939, 1947, 1949.

Nelson, K.: Structure and Strategy in Learning to Talk. Monographs of the Society for Researcb in Child Development, 1973, 38 (1-2)

Ninio, A. y BRUNER, J. S.: The achievement and antecedents of labelling. Journal of Child Language, 1978, $5,1-15$.

Osherson, D. y Markman, E. M.: Language and the ability ro evaluate contradictions and tautologies. Cognition, 1975, 3, 213-226.

Pinget, J.: Le langage et la pensée chez l'enfant. Neuchâtel, Delachaux et Niestlé. 1923.

Ronjat, J.: Le développement du langage observé chez un enfant bilingue. Paris, Champio, 1913.

SLobIN, D. I.: Cognitive prerequisites for the development of grammar, (en) C. A. Ferguson Y D. I. Slobin (eds.) Studies of Child Language Development. Nueva York, Holt, Rinehart and Winston. 1973.

SLoBin, D. I.: A case study of early language awareness, (en) A. Sinclair, . J. Jarvella Y W. J. M. Levelt (eds.) The Child's Conception Of Language. Berlin, Springer. 1978.

Swain, M.: Bilingual first language acquisition, (en) Von Raffer-Engel y S. Lebrun (eds.) Baby talk and infant speech. Amsterdam, Swets and Zeitfinger, 1976.

Tabouret-Keller, A.: Le bilinguisme de l'enfant avant six ans. Estrasburgo, Inst. de Psychologie. Univ. de Strasbourg. 1969.

Titone, R.: Bilinguisme infantile e sviluppo della personalità. Quaderni per la promozione del bilinguismo, $1980,27-28,2-22$.

Vigotsky, L. S.: Pensamiento y Lenguaje. Buenos Aires, Pléyade. 1977. (1.a ed. en ruso, 1934).

Volterra, V. y TAEsChNer, T.: The acquisition and development of language by bilingual children. Journal of Child Language, 1978, 5, 311-326.

\section{Resumen}

Este trabajo versa sobre la adquisición del lenguaje en bilingües familiares. Los datos que se aportan se refieren al léxico de uno de los sujetos de la investigación que ve recogido en sesiones de 30'grabadas en magnetoscopio, cada 2 semanas aproximadamente, a partir de los 6 basta los 24 meses de edad. Los resultados concuerdan con los de investigaciones precedentes, donde se muestra que los niños que adquieren dos lenguas a la vez pasan por una primera fase de "ssistema lexical únicon, en la cual el niño incorpora palabras de una u otra lengua, sin diferenciar los dos códigos.

\section{Résumé}

Ce travail a trait à l'acquisition du langage chez les bilingues familiales. Les données ont été obtenues a partir du lexique d'un des sujets de la recherche dont les productions furents enregistrées au magnetoscope pendant des séances de 30 minutes toutes les 2 semaines dès l'âge de 6 mois jusqu'à l'âge de 24 mois. Les résultats de notre recherche concordent sur ceux que ont été obtenus par des recherches précédentes et montrent que les enfants qui ont acquis deux langues au même temps, traversent par une première phase de usystéme lexical uniquex. oú l'enfant incorpore des mots d'une ou d'autre langue, sans en differencer les côdes. 


\section{Summary}

This paper is referred to the language acquistion of bilinguals. It shows data related to the lexic of one of the subjects of investigation. Thirty minuse sessions every two weeks were carried out from the age of six month until the subject reached the age of two years. All the sessions were videotaped. The results agree with those obtained from previous investigations, which shoued that children acquiring two languages simultaneousty, go through an initial stage in wich they use only one lexical system to which they incorporate words from either language, not differentiating the two systems. 